\title{
Modification of glucose import capacity in Escherichia coli: physiologic consequences and utility for improving DNA vaccine production
}

Laura G Fuentes ${ }^{1}$, Alvaro R Lara², Luz M Martínez', Octavio T Ramírez ${ }^{3}$, Alfredo Martínez ${ }^{1}$, Francisco Bolívar ${ }^{1}$ and Guillermo Gosset ${ }^{1,4^{*}}$

\begin{abstract}
Background: The bacterium Escherichia coli can be grown employing various carbohydrates as sole carbon and energy source. Among them, glucose affords the highest growth rate. This sugar is nowadays widely employed as raw material in industrial fermentations. When $E$. coli grows in a medium containing non-limiting concentrations of glucose, a metabolic imbalance occurs whose main consequence is acetate secretion. The production of this toxic organic acid reduces strain productivity and viability. Solutions to this problem include reducing glucose concentration by substrate feeding strategies or the generation of mutant strains with impaired glucose import capacity. In this work, a collection of $E$. coli strains with inactive genes encoding proteins involved in glucose transport where generated to determine the effects of reduced glucose import capacity on growth rate, biomass yield, acetate and production of an experimental plasmid DNA vaccine ( $\mathrm{pHN})$.
\end{abstract}

Results: A group of 15 isogenic derivatives of E. coli W3110 were generated with single and multiple deletions of genes encoding glucose, mannose, beta-glucoside, maltose and $\mathrm{N}$-acetylglucosamine components of the phosphoenolpyruvate:sugar phosphotransferase system (PTS), as well as the galactose symporter and the Mgl galactose/glucose ABC transporter. These strains were characterized by growing them in mineral salts medium supplemented with $2.5 \mathrm{~g} / \mathrm{L}$ glucose. Maximum specific rates of glucose consumption $\left(q_{s}\right)$ spanning from 1.33 to $0.32 \mathrm{~g} / \mathrm{g} \mathrm{h}$ were displayed by the group of mutants and W3110, which resulted in specific growth rates ranging from $0.65-0.18 \mathrm{~h}^{-1}$. Acetate accumulation was reduced or abolished in cultures with all mutant strains. W3110 and five selected mutant derivatives were transformed with pHN. A 3.2-fold increase in pHN yield on biomass was observed in cultures of a mutant strain with deletion of genes encoding the glucose and mannose PTS components, as well as Mgl.

Conclusions: The group of $E$. coli mutants generated in this study displayed a reduction or elimination of overflow metabolism and a linear correlation between $q_{s}$ and the maximum specific growth rate as well as the acetate production rate. By comparing DNA vaccine production parameters among some of these mutants, it was possible to identify a near-optimal glucose import rate value for this particular application. The strains employed in this study should be a useful resource for studying the effects of different predefined $q_{s}$ values on production capacity for various biotechnological products.

Keywords: Escherichia coli, Glucose transport, Phosphoenolpyruvate, Carbohydrate phosphotransferase system, DNA vaccine

\footnotetext{
* Correspondence: gosset@ibt.unam.mx

'Departamento de Ingeniería Celular y Biocatálisis, Instituto de Biotecnología, Universidad Nacional Autónoma de México, Apdo. Postal 510-3, Cuernavaca, Morelos 62210, Mexico

${ }^{4}$ Instituto de Biotecnología, Universidad Nacional Autónoma de México,

Apdo. Postal 510-3, Cuernavaca, Morelos 62210, Mexico

Full list of author information is available at the end of the article
} 


\section{Background}

Biotechnological processes with Escherichia coli strains modified for protein, DNA or metabolite production frequently employ media containing glucose [1]. This carbohydrate is widely utilized as a raw material since it is relatively inexpensive and it is the preferred carbon and energy source for $E$. coli and other industrial microorganisms. In $E$. coli, glucose is internalized into the cytoplasm by the phosphoenolpyruvate:sugar phosphotransferase system (PTS). This is a complex protein system, widespread in bacteria and absent in Archaea and eukaryotic organisms [2]. PTS also participates in the transport and phosphorylation of other carbohydrates in addition to glucose. This system is composed of the soluble and non sugarspecific protein components enzyme I (EI) and the phosphohistidine carrier protein $(\mathrm{HPr})$ that relay a phosphoryl group from phosphoenolpyruvate (PEP) to the sugar-specific enzymes IIA and IIB. PTS components IIC, and in some cases also IID, constitute integral membrane protein permeases that transports sugar molecules, which are phosphorylated by component IIB (Figure 1). In E. coli, 21 different enzyme II complexes have been identified that participate in import and phosphorylation of various carbohydrates [2]. Glucose is imported by the glucose enzyme II complex $\left(\mathrm{II}^{\mathrm{Glc}}\right.$ ) that includes the IIA ${ }^{\mathrm{Glc}}$ enzyme and the integral membrane permease $\mathrm{IICB}^{\mathrm{Glc}}$ [3]. The mannose complex (II ${ }^{\text {Man }}$ ) is composed of the IIAB $^{\text {Man }}$ enzyme and the integral membrane permease $\mathrm{IICD}^{\mathrm{Man}}$. It has been reported that in addition to mannose, this PTS complex can also transport glucose [4]. An E. coli mutant strain with inactive glucose PTS complex still displays growth on glucose with a rate corresponding to about $80 \%$ of that observed in a wild type strain [5].

When $E$. coli grows under conditions of glucose limitation, proteins normally involved in galactose transport are induced and can also import glucose [6]. This physiological response has also been observed in $E$. coli strains with inactive PTS, even when growing in nonlimiting glucose concentrations [7]. One of the genes induced under these conditions is galP, encoding the galactose: $\mathrm{H}^{+}$symporter GalP [8]. The genes $m g l A B C$, encoding an ATP-binding protein, a galactose/glucose periplasmic binding protein and an integral membrane transporter protein, respectively, are also induced under glucose limitation. These proteins constitute the highaffinity $\mathrm{ABC}$ transporter $\mathrm{Mgl}$ system, involved in galactose/glucose transport [6]. The enzyme glucokinase, encoded by $g l k$, catalyzes the ATP-dependent phosphorylation of the glucose molecules internalized by GalP or the Mgl system [9].

Under aerobic conditions when the glucose concentration in the medium is non-limiting, E. coli displays a high rate of substrate consumption and a corresponding high specific growth rate $(\mu)$. Under these conditions, $E$. coli secretes the organic acid acetate [10,11]. This is the result of a metabolic imbalance, also known as overflow metabolism, where the rate of acetyl-coenzyme A (AcCoA) synthesis surpasses the capacity of the tricarboxylic acid (TCA) cycle to completely consume this metabolite. Therefore, the amount of AcCoA not consumed by the TCA cycle is diverted into the phosphotransacetylase (Pta)-acetate kinase (Ack) pathway where acetate is

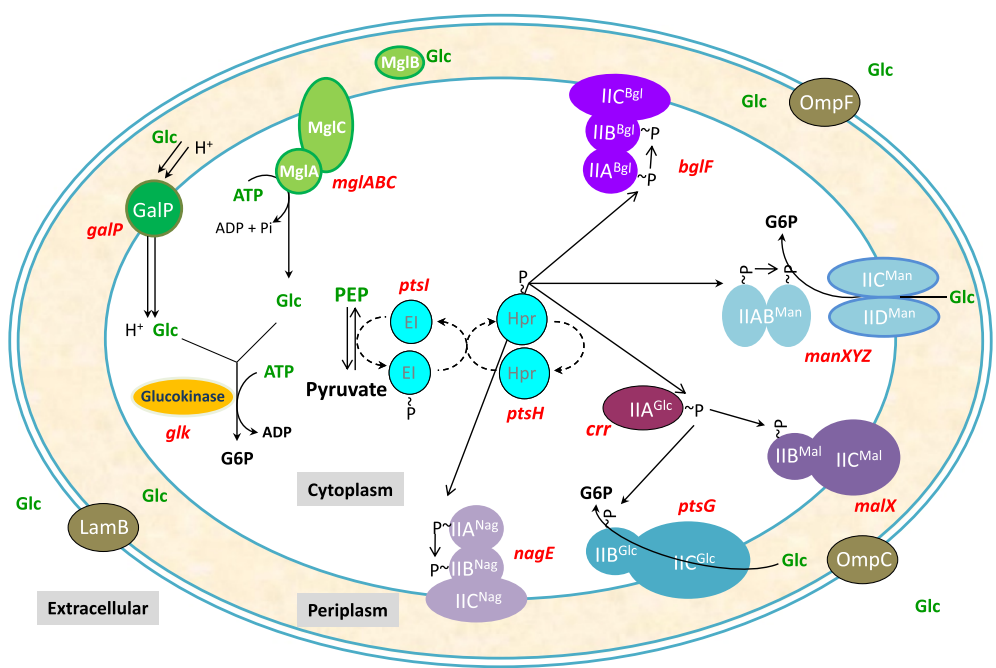

Figure 1 Proteins involved in glucose transport and phosphorylation in E. coli. El, enzyme l; HPr, phosphohistidine carrier protein; $\| C B^{G l c}$, integral membrane glucose permease; IIABC ${ }^{\mathrm{Bgl}}$, components of the beta-glucoside PTS complex; IIAB ${ }^{\mathrm{Man}}$ and IICD ${ }^{\mathrm{Man}}$ components of the mannose PTS complex; IIBC ${ }^{\mathrm{Mal}}$, components of the maltose PTS complex; IIABC ${ }^{\mathrm{Nag}}$, components of the $\mathrm{N}$-acetylglucosamine PTS complex; GalP, galactose: $\mathrm{H}^{+}$symporter; MglA, MglB and MglC, components of the galactose/glucose high-affinity $A B C$ transporter; LamB, OmpF and $O m p C$, outer membrane proteins. 
synthesized [12]. Recent studies have proposed that acetate synthesis by the Pta-Ack pathway and its assimilation by AcCoA synthethase (Acs), constitute a futile cycle that maintains a physiological level of the regulatory metabolite acetyl-phosphate. A shift towards acetate accumulation occurs as a result of carbon catabolite repression of Acs expression under conditions of high glucose import rates $[13,14]$. Acetate production is a problem since this constitutes the loss of precursor molecule AcCoA. In addition, acetate is a toxic organic acid whose accumulation in culture media reduces productivity [15]. Solutions to this problem involve glucose feeding strategies to maintain a low concentration of this sugar in culture medium and also the generation of mutant strains with impaired acetate biosynthesis capacity [16,17]. In addition, mutant strains with reduced glucose import capacity have been generated with the purpose of decreasing acetate production. Inactivation of gene $p t s G$, encoding $\mathrm{IICB}^{\mathrm{Glc}}$, resulted in a significant reduction of acetate secretion with a concomitant increase in production of a recombinant protein [5]. The complete inactivation of PTS by deletion of the ptsHI-crr operon encoding EI, HPr and IIA ${ }^{\text {Glc }}$ combined with the chromosomal overexpression of gene galP, encoding a non-PTS glucose importer, resulted in a strain displaying a $\mathrm{PTS}^{-} \mathrm{Glc}^{+}$phenotype, a lower rate of acetate production and a higher capacity for recombinant protein production [18].

One of the biotechnological products synthesized nowadays by recombinant $E$. coli is plasmid DNA (pDNA), which can be used as an alternative for gene therapy and immunization in various therapeutic applications [19]. Since plasmid titer is directly related to biomass formation, fermentation strategies for pDNA production are based on increasing cellular density. However, as stated above, acetate production can be an important drawback in this type of cultures. Some of the strategies employed to reduce acetate production in $E$. coli have been applied to pDNA production, with various degrees of success [19-21]. In the case of PTS modification, the use of a strain with a $\mathrm{PTS}^{-} \mathrm{Glc}^{+}$phenotype and reduced $q_{s}$, resulted in increased production of pDNA [20].

The above examples illustrate how a $q_{s}$ reduction in $E$. coli can have a positive effect on strain performance for the production of recombinant proteins and pDNA. However, previous works have focused on generating and studying either one or a few mutant strains with altered $q_{s}$. It remains to be determined what would be the physiological and production performance effects of altering $q_{s}$ over a wide range of values in a group of isogenic strains. With this purpose, a collection of $E$. coli mutants was generated with mutations in transporter proteins involved in glucose import. This group of 15 mutants and wild type W3110 span a $q_{s}$ range from 1.33 to $0.32 \mathrm{~g} / \mathrm{g} \mathrm{h}$, enabling a comparative study of the consequences of altering, within predetermined values, the glucose import capacity on key physiological parameters and the capacity to produce pDNA.

\section{Methods}

\section{Strains and plasmids}

The strains used in this work are described in Table 1. The reference strain E. coli W3110 is a derivative of $E$. coli K-12 [22]. Mutant derivatives of W3110 were constructed by $\mathrm{P} 1$ transduction, chromosomal gene inactivation employing PCR products or a combination of both methods [23]. Table 1 indicates the donor strains from the Keio collection that were employed as donors for P1 transduction experiments and the names of the oligonucleotides employed for PCR product gene inactivation [24]. To generate strains with more than one mutation, the gene encoding for kanamycin (Kan) resistance was excised employing plasmid pCP20 that expresses recombinase FLP [23]. Verification of the desired genotypes was made by PCR employing pairs of primers as follows: ptsG, ptsGF and ptsGR; $\operatorname{man} X$, manXF and ManXR; malX, MalXF and MalXR; $b f l F$, bglFF and bglFR; nagE, nagEF and nagER; glK, glk2F and glk2R; galP, galPF and galPR; $m g l A B C$, mglABF and mglABR; ptsHIcr, PTSF and PTSR. Plasmid pcDNAHN176-construct ( $\mathrm{pHN}$ ) is an experimental vaccine against mumps. It contains the pUC origin of replication, an ampicillin resistance gene and a viral haemagglutininneuraminidase gene cloned under transcriptional control of the cytomegalovirus promoter [25].

\section{Growth media and cultivation conditions}

The medium used during mutant strains construction and selection was Luria-Bertani (LB) with the corresponding antibiotic: chloramphenicol $(\mathrm{Cm}) 10 \mu \mathrm{g} / \mathrm{mL}$, $\mathrm{Km} 10 \mu \mathrm{g} / \mathrm{ml}$ and carbenicilin (Cb) $100 \mu \mathrm{g} / \mathrm{mL}$. For shake flask cultures M9 mineral medium was used, containing $2.5 \mathrm{~g} / \mathrm{L}$ glucose, $6 \mathrm{~g} / \mathrm{L} \mathrm{Na} \mathrm{HPO}_{4}, 0.5 \mathrm{~g} / \mathrm{L} \mathrm{NaCl}, 3 \mathrm{~g} / \mathrm{L}$ $\mathrm{KH}_{2} \mathrm{PO}_{4}, 1 \mathrm{~g} / \mathrm{L} \mathrm{NH} \mathrm{NH}_{4} \mathrm{Cl} 0.5 \mathrm{~g} / \mathrm{L} \mathrm{MgSO}_{4} \cdot 7 \mathrm{H}_{2} \mathrm{O} 0.01 \mathrm{~g} / \mathrm{L}$ $\mathrm{CaCl}_{2}$ and $0.01 \mathrm{~g} / \mathrm{L}$ thiamine hydrochloride. Cultures with strains for $\mathrm{pHN}$ production were performed in shake flasks with PD medium containing $2.5 \mathrm{~g} / \mathrm{L}$ glucose, 17 g/L K2HPO4, 5.3 g/L KH2PO4, 2.5 g/L (NH4) 2SO4, $1 \mathrm{~g} / \mathrm{L}$ (NH4)Cl, $1 \mathrm{~g} / \mathrm{L}$ sodium citrate, $1 \mathrm{~g} / \mathrm{L}$ $\mathrm{MgSO} 4 \cdot 7 \mathrm{H} 2 \mathrm{O}, 0.01 \mathrm{~g} / \mathrm{L}$ thiamine hydrochloride and 2 $\mathrm{mL}$ of a stock solution of trace elements [20]. Glucose and salts solution were sterilized separately at $121^{\circ} \mathrm{C}$ during 20 minutes. All stock cultures were stored at $-70^{\circ}$ $\mathrm{C}$ in LB medium containing glycerol (40\% v/v). The inoculum for shake flasks cultures consisted of $5 \mathrm{ml}$ from LB overnight cultures that were sub-cultured in shake flasks containing $50 \mathrm{ml}$ of M9 or PD medium with $2.5 \mathrm{~g} / \mathrm{L}$ glucose starting with an $\mathrm{OD}_{600 \mathrm{~nm}}=0.1$ and incubated at $37^{\circ} \mathrm{C}, 300 \mathrm{rpm}$ in an orbital shaker until an OD of 2.0 was reached, then, a sample was used to inoculate 
Table 1 E. coli strains and plasmid employed in this study

\begin{tabular}{|c|c|c|}
\hline Name & Description & Source or construction method* \\
\hline \multicolumn{3}{|l|}{ Strains } \\
\hline JW1087-2 & 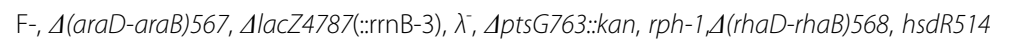 & [23] \\
\hline JW1806-1 & $\mathrm{F}-, \Delta(\operatorname{araD}-\mathrm{araB}) 567, \Delta$ lacZ4787(::rrnB-3), $\lambda, \Delta$ manX741::kan, rph-1,4(rhaD-rhaB)568, hsdR514 & {$[23]$} \\
\hline JW1613-1 & 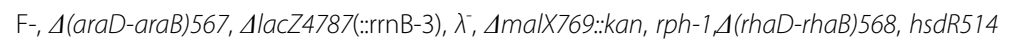 & {$[23]$} \\
\hline JW3700-1 & 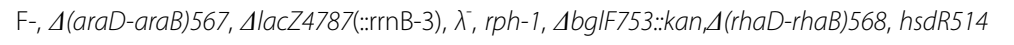 & {$[23]$} \\
\hline JW0665-1 & F-, $\Delta($ araD-araB) 567, $\Delta$ lacZ4787(::rrnB-3), $\Delta$ nagE728::kan, $\lambda$, rph-1, $($ (rhaD-rhaB) 568, hsdR514 & {$[23]$} \\
\hline JW2910-1 & 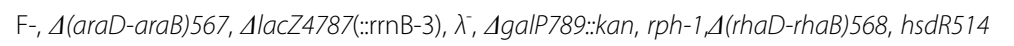 & [23] \\
\hline W3110 & $F^{-} \lambda^{-} r p h-1$ IN(rrnD-rrnE)1 & [23] \\
\hline WG & W3110 $\Delta p t s G:: F R T-K m-F R T$ & This study JW1087-2 \\
\hline WGX & WG $\triangle$ malX::FRT-Km-FRT & This study JW1613-1 \\
\hline WGB & WG $\triangle b g \mid F:: F R T-K m-F R T$ & This study JW3700-1 \\
\hline WGE & WG $\Delta$ nagE::FRT-Km-FRT & This study JW0665-1 \\
\hline WGM & WG $\Delta$ manX::FRT-Km-FRT & This study JW1806-1 \\
\hline WGMX & WGM $\Delta$ malX::FRT-Km-FRT & This study JW1613-1 \\
\hline WGMB & WGM $\Delta b g l F:: K m ; F R T-K m-F R T$ & This study JW3700-1 \\
\hline WGME & WGM $\Delta$ nagE::FRT-Km-FRT & This study JW0665-1 \\
\hline WGP & WG $\triangle$ galP::FRT-Km-FRT & This study JW2910-1 \\
\hline WGC & WG $\triangle m g \mid A B C:: F R T-C m-F R T$ & This study mglABCDtF, mglABCDtR \\
\hline WGMP & WGM $\triangle$ galP::FRT-Km-FRT & This study JW2910-1 \\
\hline WGMC & WGM, $\triangle m g I A B C:: F R T-C m-F R T$ & This study mglABCDtF, mglABCDtR \\
\hline WHI & W3110 sptsHlcrr::FRT-Cm-FRT & This study PTSDF PTSDR \\
\hline WHIP & WHI $\triangle$ galP::FRT-Km-FRT & This study JW2910-1 \\
\hline WHIC & WHI $\triangle m g \mid A B C:: F R T-C m-F R T$ & This study mglABCDtF, mglABCDtR \\
\hline W3110p & W3110/pHN & This study \\
\hline WGp & WG/pHN & This study \\
\hline WGMCp & WGMAB/pHN & This study \\
\hline WGMp & WGM/pHN & This study \\
\hline WGMEp & WGME/pHN & This study \\
\hline WHICp & WHIAB/pHN & This study \\
\hline \multicolumn{3}{|l|}{ Plasmids } \\
\hline $\mathrm{pHN}$ & $\begin{array}{l}\text { Derivative of pcDNA3.1 with cloned gene encoding for a } 567 \text { nucleotide region from } \\
\text { the HN gene }\end{array}$ & {$[25]$} \\
\hline pKD3 & bla, FRT-Cm-FRT & {$[23]$} \\
\hline pKD46 & bla, $>\beta$ exo (red recombinase), temperature-conditional replicon & [23] \\
\hline pCP20 & bla, flp, temperature-conditional replicon & [23] \\
\hline \multicolumn{3}{|l|}{ Primers } \\
\hline ptsGF & CGC AGG TAA CCA CCG ATA AC & This study \\
\hline ptsGR & GCA ACG CGC TAT ATT GCA GA & This study \\
\hline $\operatorname{manXF}$ & ATC TGG CAC GTT GAC GTG TT & This study \\
\hline $\operatorname{man} X R$ & TTG CCG TTA TCA GCA GCC TT & This study \\
\hline malXF & AGC CAT GCA GAT GAC CTA CT & This study \\
\hline malXR & AAC GGT CAG CGA CAT AAT CC & This study \\
\hline bglFF & GGA TTG TTA CCG CAC TAA GC & This study \\
\hline bglFR & AGG CAC CTT CCA CCT GAT TG & This study \\
\hline nagEF & CGT TGG CGG ATT AGG CAT CT & This study \\
\hline
\end{tabular}


Table $1 E$. coli strains and plasmid employed in this study (Continued)

\begin{tabular}{|c|c|c|}
\hline nagER & TGT TGG ATG CGA CGC TCA AG & This study \\
\hline glk2F & CCG CCA GCA AGA CCG AGA AT & This study \\
\hline glk2R & TCT ACC GCC GCT TCT TCC AG & This study \\
\hline galPF & CGA TGC TGC CGG TCT GAA GT & This study \\
\hline galPR & GTG TTG CGA CGC ACG GAT TG & This study \\
\hline PTSF & CGA TGT GGC GGT AAC AAT CT & This study \\
\hline PTSR & CCG CTT CAT AGC AGG TAT GT & This study \\
\hline mglABF & GCT TCG GCG TTC AGT AAC AC & This study \\
\hline mglABR & TAT GAC CGA ATG CGG ACC AC & This study \\
\hline PTSDF & $\begin{array}{l}\text { CTA GAC TाT AGT TCC ACA ACA CTA AAC CTA TAA GTT GGG GAA ATA CAA TGG TGT } \\
\text { AGG CTG GAG CTG CTT C }\end{array}$ & This study \\
\hline PTSDR & $\begin{array}{l}\text { ATG GGC GCC ATT TाT CAC TGC GGC AAG AAT TAC TTC TTG ATG CGG ATA ACA TGG } \\
\text { GAA TTA GCC ATG GTC C }\end{array}$ & This study \\
\hline mglABCDtF & $\begin{array}{l}\text { AGC ATT TAT CTC AAG CAC TAC CCT GCA TAA GAA AAA CCG GAG ATA CCA TGG TGT } \\
\text { AGG CTG GAG CTG CTT C }\end{array}$ & This study \\
\hline mglABCDtR & $\begin{array}{l}\text { TाT ATG ACC GAA TGC GGA CCA CAT TCA CAT CAT TTC TTA CGC GCG TAT TTA TGG } \\
\text { GAA TTA GCC ATG GTC C }\end{array}$ & This study \\
\hline
\end{tabular}

*The donor strain employed in P1 transduction or the names of the oligonucleotides employed for gene inactivation with PCR products are indicated.

shake flasks containing $50 \mathrm{~mL}$ of the same medium. Samples for analysis were taken periodically. The $\mathrm{OD}_{600 \mathrm{~nm}}$ was determined and cells were centrifuged $(13,000 \mathrm{~g}$ for $5 \mathrm{~min})$ and the supernatant used for glucose and acetate determination by HPLC. In cultures for $\mathrm{pHN}$ production, an additional sample was taken to determine plasmid DNA concentration. The employed $\mathrm{OD}_{600 \mathrm{~nm}}$ and cell dry mass $(\mathrm{CDM})(\mathrm{g} / \mathrm{L})$ correlation was $\mathrm{CDM}=0.45 \times \mathrm{OD}_{600 \mathrm{~nm}}$ [26].

\section{Determination of glucose, acetic acid and plasmid concentration}

The concentration of D-glucose and organic acids was determined employing an Aminex HPX-87H column (300 X 7.8 mm; 9 Am Bio-Rad, Hercules, CA). Separation was carried out isocratically with $5 \mathrm{mM} \mathrm{H}_{2} \mathrm{SO}_{4}$ at a flow rate of $0.5 \mathrm{~mL} / \mathrm{min}$ and a temperature of $50^{\circ} \mathrm{C}$. Under these conditions glucose was detected by refraction index and acetic acid by photodiode array at 210 $\mathrm{nm}$. For these measurements a Waters HPLC system was used: 600E quaternary pump, 717 automatic injector, 2410 refraction index, and 996 photodiode array. pHN concentration was determined by extracting plasmid DNA with the Qiagen Spin Mini Prep kit (Hilden, Germany), following manufacturer instructions. The plasmid DNA concentration was determined at $260 \mathrm{~nm}$ using a Nanodrop UV spectrophotometer [27].

\section{Kinetic parameters calculation}

For the characterization of the strains used in this work, maximum specific rates of growth $(\mu)$, glucose consumption $\left(q_{s}\right)$, acetate production $\left(q_{a c}\right)$, pHN plasmid production $\left(q_{p}\right)$, yield of biomass on glucose $\left(\mathrm{Y}_{\mathrm{X} / \mathrm{S}}\right)$, yield of acetate on glucose $\left(\mathrm{Y}_{\mathrm{ac} / \mathrm{S}}\right)$, yield of acetate on biomass $\left(\mathrm{Y}_{\mathrm{ac} / \mathrm{X}}\right)$, yield of plasmid on glucose $\left(\mathrm{Y}_{\mathrm{P} / \mathrm{S}}\right)$ and yield of plasmid on biomass $\left(\mathrm{Y}_{\mathrm{P} / \mathrm{X}}\right)$ were determined. Yield values were calculated using linear regression of product concentration versus substrate or biomass concentration during the exponential growth phase. The linear least squares fit to the data displayed $R^{2}$ values equal or higher than 0.97 .

Values for $q_{s}, q_{a c}$, and $q_{p}$ were calculated employing the following equations:

$$
\begin{gathered}
q_{s}=m \mu / \mathrm{Yxs} \\
q_{a c}=\mathrm{Y}_{\mathrm{ac} / \mathrm{X}} \cdot \mu \\
q_{p}=\mathrm{Y}_{\mathrm{P} / \mathrm{X}} \cdot \mu
\end{gathered}
$$

These parameters were calculated during exponential growth phase. Flask cultures were performed in triplicate and the values reported represent the mean of all experiments.

\section{Results and discussion}

Generation and characterization of $E$. coli mutant strains lacking proteins involved in glucose import

The aim of this study was to generate and characterize a group of mutants derived from strain W3110, displaying a wide range of $q_{s}$ values. The strategy followed to progressively decrease glucose import capacity consisted on inactivating genes encoding PTS components and non-PTS transport proteins. Under the growth conditions employed in this study (glucose $2.5 \mathrm{~g} / \mathrm{L}$ ), it can be expected that glucose is transported by the PTS, since the $K_{M}$ of $\mathrm{IICB}^{\mathrm{Glc}}$ for this sugar is 3 to $10 \mu \mathrm{M}$ (0.6 to $18 \mathrm{mg} / \mathrm{L})$ [3]. Therefore, mutants with inactive $\mathrm{IICB}^{\text {Glc }}$ and other PTS components 
were generated and characterized. It has been reported that $\mathrm{IICD}^{\mathrm{Man}}$ can transport glucose in a strain lacking $\mathrm{IICB}^{\mathrm{Glc}}$ activity [4]. However, it is not known which of the other remaining PTS complexes could be involved in glucose transport in the absence of $\mathrm{IICB}^{\mathrm{Glc}}$ or in a double mutant lacking both $\mathrm{IICB}^{\text {Glc }}$ and IICD ${ }^{\text {Man }}$ components. In this study, we chose to inactivate selected components from the glucose and mannose PTS families, assuming they would show specificity towards glucose as substrate since both, IICB ${ }^{\text {Glc }}$ and IICD ${ }^{\text {Man }}$, can transport this sugar. Since the number of PTS complexes belonging to these two families is rather large, only a few members were arbitrarily selected for inactivation. Single, double and triple mutant derivatives of W3110 were constructed including genes encoding transport proteins from the glucose (ptsG), mannose, $(\operatorname{man} X)$, beta-glucoside $(b g l F)$, maltose (malX) and N-acetylglucosamine (nagE) PTS complexes. Inactivation of ptsG (strain WG) caused a $21 \%, 22 \%$ and $63 \%$ reduction in $q_{s}, \mu$ and $q_{a c}$, respectively (Table 2 and Additional file 1: Figure S1). This is expected since IICB ${ }^{\text {Glc }}$ is the main glucose importer under the studied conditions. However, these results also show that there is still significant remaining glucose import capacity in the mutant strain; therefore, other transport proteins are involved in this process. To generate mutant derivatives of strain WG with further reduced $q_{s}$, genes encoding specific components of the above-mentioned PTS complexes were inactivated. In the pts $G^{-}$background, inactivation of malX (strain WGX), nagE (strain WGE) or manX (strain WGM) caused a further reduction in $q_{s}$ and $\mu$. Furthermore, no acetate was detected in the supernatants of cultures with these three strains, indicating the elimination of overflow metabolism (Additional file 1: Figure S1). In contrast, inactivation of ptsG and $b g l F$ (strain WGB) did not decrease $q_{s}$, when compared to strain WG. These results indicate that mannose, maltose and $\mathrm{N}$-acetylglucosamine PTS complexes contribute to glucose import in the absence of $\mathrm{IICB}^{\mathrm{Glc}}$. From strain WGM, mutant derivatives were generated to determine if beta-glucoside, maltose or $\mathrm{N}$ acetylglucosamine PTS complexes participate in glucose transport in this genetic background. From these mutant strains, only WGME showed a reduction in $q_{s}$ and $\mu$ of $58 \%$ and 57\%, respectively, when compared to W3110.

Previous results indicated that strain WGME displays growth on glucose even after the inactivation of several transport proteins. Glucose transport in this strain could be dependent on both PTS and non-PTS transport proteins. To determine the extent of the contribution to glucose transport of the still-active PTS complexes in strain WGME, a mutant derivative of W3110 was generated by deletion of the ptsHI, -crr operon (strain WHI). Deletion of genes encoding EI and HPr disrupts the phosphotransfer chain, thus rendering all PTS complexes inactive. As expected, the complete elimination of PTS activity caused a severe reduction in $q_{s}$ and $\mu$ of $64 \%$ and $62 \%$, respectively, when compared to W3110. When compared to WGME, strain WHI showed $12 \%$ and $14 \%$ reduction in $\mu$ and $q_{s}$, respectively. These results suggest that approximately $14 \%$ of the glucose import capacity in WGME is dependent on one or several of the still active PTS complexes in this strain, and the remaining capacity is dependent on non-PTS transporters. However, this conclusion should be taken with caution, since PTS is involved in regulatory processes

Table 2 Kinetic and stoichiometric parameters of wild type and mutant strains lacking various glucose transporters

\begin{tabular}{|c|c|c|c|c|c|}
\hline Strains & Deleted gene(s) & $\mu\left(\mathrm{h}^{-1}\right)$ & $q_{s}(\mathrm{~g} / \mathrm{g} \mathrm{h})$ & $q_{a c}(g / g h)$ & $\mathrm{Y}_{\mathrm{X} / \mathrm{S}}$ \\
\hline W3110 & - & $0.65 \pm 0.02$ & $1.33 \pm 0.04$ & $0.19 \pm 0.02$ & $0.49 \pm 0.00$ \\
\hline WG & $p t s G$ & $0.51 \pm 0.01$ & $1.05 \pm 0.12$ & $0.07 \pm 0.05$ & $0.53 \pm 0.12$ \\
\hline WGX & ptsG, malX & $0.23 \pm 0.03$ & $0.51 \pm 0.01$ & 0 & $0.41 \pm 0.01$ \\
\hline WGB & $p t s G, b g l F$ & $0.43 \pm 0.04$ & $1.06 \pm 0.03$ & $0.06 \pm 0.02$ & $0.42 \pm 0.03$ \\
\hline WGE & ptsG, nage & $0.41 \pm 0.02$ & $0.69 \pm 0.06$ & 0 & $0.60 \pm 0.08$ \\
\hline WGM & ptsG, manX & $0.36 \pm 0.03$ & $0.65 \pm 0.05$ & 0 & $0.56 \pm 0.08$ \\
\hline WGMX & ptsG, manX, malX & $0.32 \pm 0.01$ & $0.58 \pm 0.06$ & 0 & $0.55 \pm 0.06$ \\
\hline WGMB & $p t s G, \operatorname{man} X$, bglF & $0.29 \pm 0.00$ & $0.66 \pm 0.07$ & 0 & $0.40 \pm 0.02$ \\
\hline WGME & ptsG, manX, nagE & $0.28 \pm 0.02$ & $0.56 \pm 0.00$ & 0 & $0.48 \pm 0.03$ \\
\hline WGP & ptsG, galP & $0.49 \pm 0.08$ & $1.19 \pm 0.00$ & $0.06 \pm 0.00$ & $0.44 \pm 0.01$ \\
\hline WGC & $p t s G, m g \mid A B C$ & $0.48 \pm 0.01$ & $1.02 \pm 0.00$ & $0.08 \pm 0.00$ & $0.47 \pm 0.05$ \\
\hline WGMP & ptsG, manX, galP & $0.29 \pm 0.01$ & $0.56 \pm 0.01$ & 0 & $0.52 \pm 0.02$ \\
\hline WGMC & $p t s G, \operatorname{man} X, \operatorname{mg} \mid A B C$ & $0.31 \pm 0.01$ & $0.68 \pm 0.00$ & 0 & $0.47 \pm 0.01$ \\
\hline WHI & ptsHIcrr & $0.25 \pm 0.01$ & $0.49 \pm 0.03$ & 0 & $0.51 \pm 0.02$ \\
\hline WHIP & ptsHlcrr, galP & $0.18 \pm 0.02$ & $0.32 \pm 0.02$ & 0 & $0.55 \pm 0.03$ \\
\hline WHIC & ptsHIcrr, mglABC & $0.20 \pm 0.01$ & $0.32 \pm 0.02$ & 0 & $0.38 \pm 0.05$ \\
\hline
\end{tabular}


that include genes encoding non-PTS transporters [28]. Therefore, it cannot be ruled out that altered regulatory responses on genes encoding non-PTS transporters could occur in strain WHI, when compared to WGME.

The transporter GalP and the Mgl protein system are known to participate in glucose import under conditions of glucose starvation. Even though the culture medium employed in this study contains a non-limiting glucose concentration, as a result of the inactivation of glucose importers, a low intracellular level of glucose-6-phosphate and other central metabolism intermediates could be expected in the mutant strains displaying low $q_{s}$ values. To determine if GalP or Mgl are involved in glucose transport in some of these strains, derivatives lacking either one of these two importers were generated from WG and WGM. Strains WGP and WGC showed similar $q_{s}$ and $\mu$ values as progenitor strain WG, indicating that GalP or Mgl do not contribute to glucose import in this genetic background. Whereas in the WGM background, strain WGMC did not show a change in glucose import capacity, while mutant WGMP displayed a decrease in $q_{s}$ and $\mu$. These results show that only after the $q_{s}$ decreases to a value close to $0.65 \mathrm{~g} / \mathrm{g} \mathrm{h}$, then a contribution of GalP to glucose import can be detected. To determine the involvement of non-PTS transporters on glucose import capacity in strain WHI, derivatives with inactive galP (WHIP) or mglABC (WHIC) were generated and characterized. Both mutants displayed a $35 \%$ reduction in $q_{s}$ when compared to progenitor WHI. These results indicate that both GalP and the Mgl system contribute to glucose import in the strain lacking functional PTS.

When comparing the $Y_{X / S}$ values among wild type and mutant strains, minimal differences were detected. The group of strains characterized in this study, including wild-type W3110, display a wide range of $q_{s}, \mu$ and $q_{a c}$ values. Under the conditions employed here, $\mu$

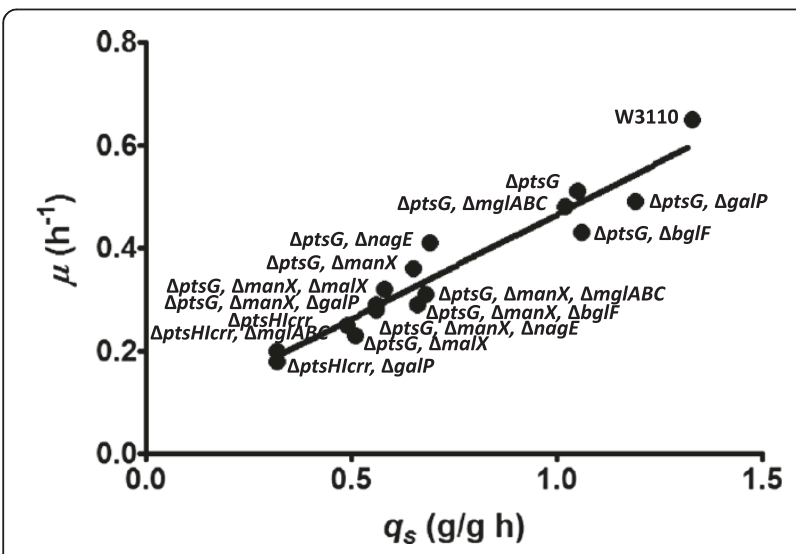

Figure 2 Specific growth rate as a function of the specific glucose uptake rate for various $E$. coli strains generated in this study. correlated linearly with $q_{s}\left(R^{2}=0.92\right)$ (Figure 2$)$. This result is consistent with the small differences in $Y_{X / S}$ among strains. As result of the distinct $q_{s}$ values displayed by these strains, the amount of time taken to completely consume the $2.5 \mathrm{~g} / \mathrm{L}$ of glucose in the culture medium varied from 6 to $28 \mathrm{~h}$ (Additional file 1: Figure S1). In addition to the physiological effects of such glucose consumption rates, these differences would be expected to have an impact on strain production capacity, mainly specific productivity, for various biotechnological products, as will be discussed below.

When considering data from the five strains that accumulated acetate in the medium (W3110, WG, WGB, WGP and WGC) and WGE, the mutant strain with the highest $q_{s}$ and $\mu$ values that did not produce acetate, it was found that $q_{a c}$ correlated linearly with $\mu\left(R^{2}=0.91\right)$ (Figure 3$)$. It is not possible to ascertain if the observed correlation is maintained at $\mu$ values lower than $0.43 \mathrm{~h}^{-1}$ (strain WGB), nevertheless, linear regression analysis of this data was employed to calculate $\mu=0.39 \mathrm{~h}^{-1}$ as the value corresponding to a $q_{a c}=0$. This value is close to a $\mu$ of $0.375 \mathrm{~h}^{-1}$, that was determined from glucose-limited continuous cultures with $E$. coli $\mathrm{K}-12 \mathrm{TG} 1$, as the lowest value where acetate was detected [29]. A study employing accelerostat cultivations with strain MG1655 identified a two-phase acetate accumulation pattern [13]. The slow rate acetate accumulation phase started at a $\mu$ of $0.27 \mathrm{~h}^{-1}$, whereas high rate acetate accumulation was detected at a $\mu$ of $0.46 \mathrm{~h}^{-1}$. Assuming a similar physiological response between strains MG1655 and W3110, it is possible that acetate accumulation in this study with mutant derivatives was detected until the high rate production phase was reached. Further detailed analyses, employing accelerostat or chemostat cultivations, should allow determination of the specific acetate accumulation pattern for each mutant strain.

The qualitative effects on $q_{s}$ and $\mu$ of deleting specific PTS and non-PTS transporters in strain W3110 are in

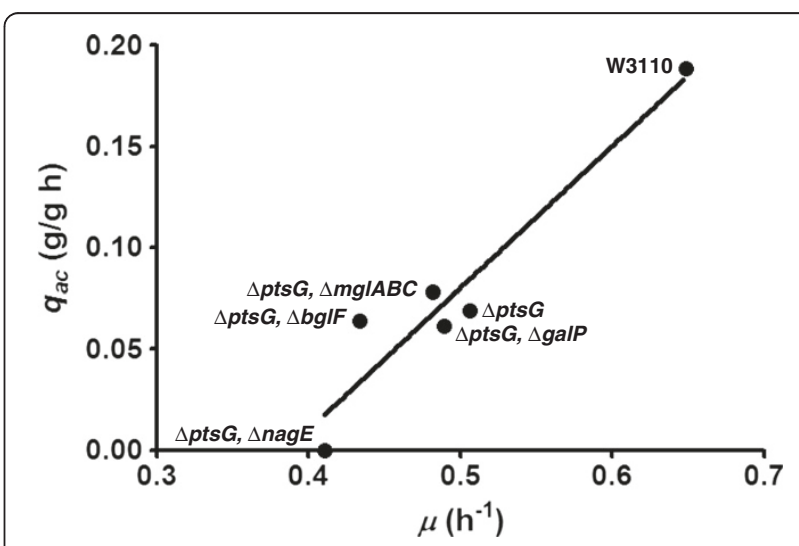

Figure 3 Specific acetate production rate as a function of the specific growth rate for various $E$. coli strains generated in this study. 
agreement with results reported with mutant derivatives of E. coli strains MG1655 and AF1000 having some of the same gene deletions performed in this study [30-32]. However, the magnitude of $q_{s}$ reduction as function of specific gene inactivation differs from our results. This can be explained considering that different culture media was employed and the strains genetic backgrounds are not identical.

\section{Generation and characterization of strains with modified glucose import capacity for the production of a DNA vaccine}

The characterization of the strains generated in this study enabled the identification of mutant derivatives of W3110 with distinct $q_{s}$, and $q_{a c}$ values. In several of these strains, acetate production was reduced or absent. Lower acetate production and accumulation is associated with an increased capacity for pDNA production in E. coli cultures $[19,20]$. To determine the effect of different $q_{s}, q_{a c}$ and $\mu$ values on pDNA production capacity, some of the strains generated in this study were transformed with plasmid pHN. Strains W3110, WG, WGM, WGME, WGMC, WHI and WHIC were transformed with plasmid $\mathrm{pHN}$ to generate recombinant strains W3110p, WGp, WGMp, WGMEp, WGMCp, WHIp and WHICp, respectively. These strains were grown in shake-flask cultures with PD medium supplemented with $2.5 \mathrm{~g} / \mathrm{L}$ glucose. This culture medium has been employed previously for pDNA production studies; therefore, it was used here to facilitate strain performance comparisons. During strain characterization, it was determined that strain WHIp did not grow in PD medium; therefore, it was not considered for further analysis. Table 3 summarizes the kinetic and stoichiometric parameters of the six remaining strains. Among these strains, acetate was detected only in cultures with W3110 $(0.4 \mathrm{~g} / \mathrm{L})$ (Additional file 2: Figure S2). The maximum
pHN titer level varied among strains, with WGMCp displaying the highest value. The same trend was observed for plasmid yield from glucose $\left(\mathrm{Y}_{\mathrm{P} / \mathrm{S}}\right)$ or from biomass $\left(\mathrm{Y}_{\mathrm{P} /}\right.$ $\mathrm{X})$. The highest plasmid specific productivity was observed for strain WGMCp and also for WGMp. Figure 4 shows the $\mathrm{Y}_{\mathrm{P} / \mathrm{X}}$ as a function of $q_{s}$ for the $\mathrm{pHN}$ producing strains. Compared to strain W3110p, the $\mathrm{Y}_{\mathrm{P} / \mathrm{X}}$ for strains WGp and WGMCp were 1.4 and 3.2-fold higher, respectively. In the case of strain WGp, the observed increase can be explained by the lack of acetate production, when compared to W3110p. A similar result has been reported when comparing wild type and mutants defective in glucose transport in the case of recombinant protein production $[18,31,33]$. The final acetate titer in cultures with W3110p was not high enough to expect a toxic effect [15]. Therefore, the increase in $\mathrm{pHN}$ production in strain WGp can be attributed mainly to the elimination of overflow metabolism, resulting in a more efficient use of the carbon source. However, the higher $\mathrm{Y}_{\mathrm{P} / \mathrm{X}}$ observed for WGMCp, when compared to WGp, cannot be explained by lower acetate production, since this metabolite was not detected in culture supernatants of either strain. Alterations to glucose consumption, either by the inactivation of glucose importers or feeding strategies to limit this nutrient, have been known to alter central carbon metabolism fluxes $[26,34]$. It can be speculated that metabolic flux distribution in WGMCp favors the synthesis of DNA precursors, leading to higher $\mathrm{pHN}$ production. A more detailed comparative analysis of these strains, including fluxome and other "omics" approaches should allow a better understanding of the observed results.

A recent study has determined $\mathrm{pHN}$ plasmid productivity with strain $\mathrm{DH} 5 \alpha$, a high-performance plasmid production strain, under culture conditions similar to those employed here. This report revealed an $\mathrm{Y}_{\mathrm{P} / \mathrm{X}}$ of $6.56 \pm$ $0.17 \mathrm{mg} / \mathrm{g}$ and a $q_{p}$ of $2.59 \pm 0.12(\mathrm{~g} / \mathrm{g} \mathrm{h})$ for $\mathrm{pHN}$ [27].

Table 3 Kinetic and stoichiometric parameters of wild type and mutant strains transformed with plasmid pHN

\begin{tabular}{|c|c|c|c|c|c|c|}
\hline Parameter & W3110p & $\begin{array}{c}\text { WGp } \\
(\Delta p t s G)\end{array}$ & $\begin{array}{c}\text { WGMCp } \\
(\Delta p t s G, \Delta \text { manX } \\
\Delta \text { mglABC })\end{array}$ & $\begin{array}{c}\text { WGMp } \\
(\Delta p t s G, \Delta \operatorname{man} X)\end{array}$ & $\begin{array}{c}\text { WGMEp } \\
(\Delta p t s G, \Delta \text { manX, } \Delta \text { nagE) }\end{array}$ & $\begin{array}{c}\text { WHICp } \\
(\Delta p t s H I c r r, \Delta m g I A B C)\end{array}$ \\
\hline $\begin{array}{l}\text { Maximum biomass } \\
\text { concentration ( } \mathrm{g} / \mathrm{L})\end{array}$ & $1.14 \pm 0.15$ & $1.42 \pm 0.02$ & $0.82 \pm 0.30$ & $1.08 \pm 0.05$ & $1.12 \pm 0.06$ & $1.13 \pm 0.16$ \\
\hline $\begin{array}{l}\text { Maximum plasmid pHN } \\
\text { concentration (mg/L) }\end{array}$ & $0.83 \pm 0.18$ & $1.06 \pm 0.19$ & $1.63 \pm 0.45$ & $1.09 \pm 0.34$ & $1.19 \pm 0.41$ & $1.47 \pm 0.30$ \\
\hline $\begin{array}{l}\text { Maximum acetate } \\
\text { concentration (g/L) }\end{array}$ & $0.40 \pm 0.01$ & 0 & 0 & 0 & 0 & 0 \\
\hline$\mu\left(h^{-1}\right)$ & $0.63 \pm 0.04$ & $0.49 \pm 0.00$ & $0.33 \pm 0.06$ & $0.33 \pm 0.02$ & $0.37 \pm 0.01$ & $0.14 \pm 0.02$ \\
\hline$Y_{p / S}(m g / g)$ & $0.43 \pm 0.15$ & $0.54 \pm 0.10$ & $1.03 \pm 0.01$ & $0.89 \pm 0.00$ & $0.75 \pm 0.05$ & $0.84 \pm 0.04$ \\
\hline$Y_{p / x}(\mathrm{mg} / \mathrm{g})$ & $0.65 \pm 0.04$ & $0.93 \pm 0.14$ & $2.10 \pm 0.30$ & $1.57 \pm 0.14$ & $1.39 \pm 0.09$ & $1.08 \pm 0.13$ \\
\hline$q_{s}(\mathrm{~g} / \mathrm{g} \mathrm{h})$ & $1.20 \pm 0.07$ & $0.89 \pm 0.07$ & $0.70 \pm 0.09$ & $0.59 \pm 0.03$ & $0.57 \pm 0.03$ & $0.31 \pm 0.07$ \\
\hline$q_{p}(\mathrm{~g} / \mathrm{g} \mathrm{h})$ & $0.50 \pm 0.09$ & $0.43 \pm 0.02$ & $0.53 \pm 0.09$ & $0.53 \pm 0.05$ & $0.51 \pm 0.02$ & $0.15 \pm 0.01$ \\
\hline Culture time(h) & 5 & 7 & 9 & 8 & 9 & 24 \\
\hline
\end{tabular}




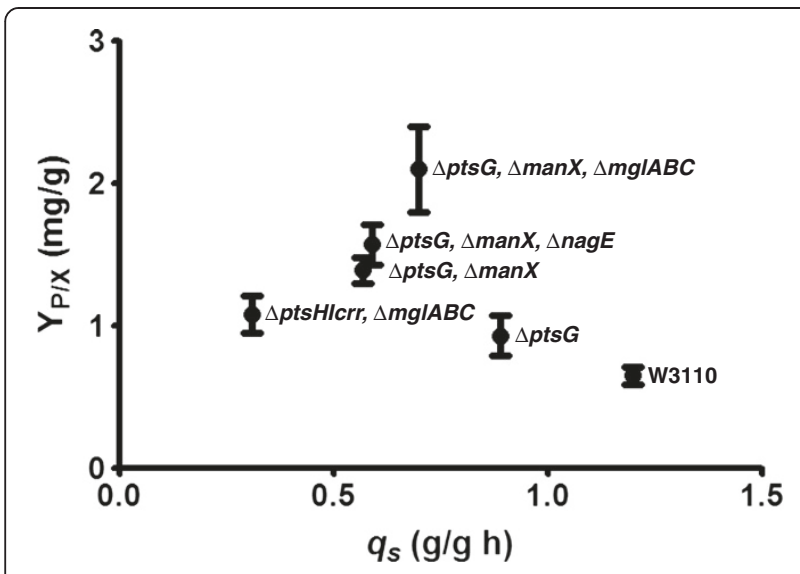

Figure 4 Plasmid pHN yield from biomass as a function of the specific glucose uptake rate for various $E$. coli strains generated in this study.

These values are approximately 3 and 5 -fold higher for $\mathrm{Y}_{\mathrm{P} / \mathrm{X}}$ and $q_{p}$, respectively, when compared to results with strain WGMCp. It should be pointed out, though, that strain $\mathrm{DH} 5 \alpha$ bears several mutations that have the effect of increasing plasmid yield and productivity. It can be expected that these mutations would also increase plasmid production capacity in strain WGMCp, although the magnitude of such improvement cannot be estimated and would have to be determined experimentally.

Compared to WGMCp, strains with progressively lower $q_{s}$, WGMp, WGMEp and WHICp displayed a lower $\mathrm{Y}_{\mathrm{P} / \mathrm{X}}$. It can be assumed that a low glucose consumption rate should cause a limitation of central carbon metabolism precursors and energy, having a negative impact on pHN production. A similar result has been reported when comparing two mutant strains for the production of a recombinant protein. A $\Delta p t s G \Delta \operatorname{man} X$ mutant produced less acetate than a $\Delta p t s G$ mutant, but unexpectedly, also less recombinant protein [33]. In this case, the authors point out that not only is low acetate production important for strain performance, but also an adequate cellular energy status.

The control of plasmid replication is a complex process influenced by the cell physiologic state. Growth rate is one of the parameters that have an impact on plasmid copy number. For plasmids having a pUC origin of replication, such as pHN, a higher copy number is observed when comparing slow to fast growing cells [35]. It has been generally accepted that plasmid copy number in inversely proportional to the growth rate [19]. By altering glucose import capacity in the studied strains, cellular parameters such as $q_{s}, q_{a c}$ and $\mu \mu$ were simultaneously modified. All of these parameters influence pHN cellular content. The $q_{s}$ and $\mu$ displayed by strain WGMCp would represent optimal or near-optimal values for $\mathrm{pHN}$ production under the studied conditions which supports the idea that there should be an optimal flux distribution for pDNA synthesis, and that it may be reached not necessarily at very low growth rates. The physiologic state in this strain would be favorable for $\mathrm{pHN}$ production by having a relatively low $\mu$ that increases plasmid content and sufficient glucose import capacity to avoid carbon and energy limitation. When compared to WGMCp, strains WGMp, WGMEp and WHICp, have lower $q_{s}$. Therefore, it is likely that these strains are energy and carbon-limited, and this state limits plasmid production capacity. A low energy state, resulting from a very low $q_{s}$ might also explain why strain WHI, when transformed with $\mathrm{pHN}$, did not display growth in minimal medium. This is likely the result of incapacity to cope with the additional demand for precursors and energy required for plasmid replication.

Previous publications have demonstrated an increased capacity for plasmid or recombinant protein production in strains having reduced glucose import capacity. However, in these reports only a few mutant strains were characterized. Therefore, only a small fraction of the potential phenotypic space was sampled. In contrast, in this study, a group of mutants was generated, covering a wide range of glucose import capacity in relatively small steps. By employing this collection of mutants, it was possible to identify a near-optimal glucose import rate for plasmid production capacity under the employed conditions. This $q_{s}$ value is associated to a physiological state were no overflow metabolism is present and the rate of glucose import still provides sufficient energy and metabolic precursors for cell growth requirements plus the burden of plasmid replication. To our knowledge, there is still no theoretical method to predict such a $q_{s}$ value, therefore, a combinatorial approach, like the one employed here, should be useful for correlating glucose consumption rate to several physiological and production parameters.

\section{Conclusions}

A group of mutants with modified glucose import capacity was derived from $E$. coli W3110, a robust strain that has been employed as production host for various biotechnological products. This group of mutants affords a large degree of phenotypic diversity that can be explored when searching for specific genotypes leading to improved production capacity. The mutant strains generated and characterized here were employed in experiments for determining the correlation of distinct $q_{s}$ values to specific physiological and pDNA vaccine production parameters. Although similar data could be obtained by employing chemostat cultures, the use of mutant strains with modified glucose import capacity should simplify subsequent production processes by enabling constant glucose consumption rates in batch cultures.

The characterization of a subset of the generated mutants allowed the identification of a near-optimal $q_{s}$ 
value for pHN production. A similar approach can be followed, employing this mutant collection, to identify phenotype(s) leading to the best production parameters for other products such as recombinant proteins.

\section{Additional files}

Additional file 1: Figure S1. Growth profile of W3110 and mutant derivatives in shake flask cultures. Glucose concentration (red squares), biomass concentration (blue circles), acetate concentration (green triangles). A. W3110, B. WG, C. WGX, D. WGB, E. WGE, F. WGM, G. WGMX, H. WGMB, I. WGME, J. WGP, K. WGC, L. WGMP, M. WGMC, N. WHI, O. WHIP, P. WHIC

Additional file 2: Figure S2. Growth profile of W3110 and mutant derivatives transformed with plasmid $\mathrm{pHN}$ in shake flask cultures. Glucose concentration (red squares), biomass concentration (blue circles), acetate concentration (green triangles). A. W3110p, B. WGp, C. WGMCp, D. WGMEp, E. WGMp, F. WHICp.

\section{Competing interests}

The authors declare that they have no competing interests.

\section{Authors' contributions}

$L F, A M$ and GG participated in the design of this study. LF and LM constructed the strains. LF realized the batch cultures and analyzed the experimental data. LF, AL, OR, AM, FB and GG participated in the analysis of the results as well as in writing and critical review of the manuscript. All authors have read and approved the manuscript.

\section{Acknowledgements}

This work was supported by CONACyT grant 177568. LGF was supported by a fellowship from CONACYT. We thank Georgina Hernández-Chávez and Mercedes Enzaldo for technical assistance and Santiago Becerra Ramírez for oligonucleotide synthesis.

\section{Author details}

'Departamento de Ingeniería Celular y Biocatálisis, Instituto de Biotecnología, Universidad Nacional Autónoma de México, Apdo. Postal 510-3, Cuernavaca, Morelos 62210, Mexico. ${ }^{2}$ Departamento de Procesos y Tecnología, Universidad Autónoma Metropolitana-Cuajimalpa. Artificios No. 40, Col. Miguel Hidalgo, Del. Álvaro Obregón, México, DF CP 01120, Mexico. ${ }^{3}$ Departamento de Medicina Molecular y Bioprocesos, Instituto de Biotecnología, Universidad Nacional Autónoma de México, Apdo. Postal 510-3, Cuernavaca, Morelos 62210, Mexico. ${ }^{4}$ Instituto de Biotecnología, Universidad Nacional Autónoma de México, Apdo. Postal 510-3, Cuernavaca, Morelos 62210, Mexico.

Received: 13 March 2013 Accepted: 26 April 2013

Published: 2 May 2013

\section{References}

1. Cameron DC, Tong IT: Cellular and metabolic engineering. An overview. Appl Biochem Biotechnol 1993, 38:105-140.

2. Tchieu JH, Norris V, Edwards JS, Saier MH Jr: The complete phosphotranferase system in Escherichia coli. J Mol Microbiol Biotechnol 2001, 3:329-346.

3. Misset O, Blaauw M, Postma PW, Robillard GT: Bacterial phosphoenolpyruvate-dependent phosphotransferase system, Mechanism of the transmembrane sugar translocation and phosphorylation. Biochemistry 1983, 22:6163-6170.

4. Curtis SJ, Epstein W: Phosphorylation of D-glucose in Escherichia coli mutants defective in glucosephosphotransferase, mannosephosphotransferase, and glucokinase. J Bacteriol 1975, 122:1189-1199.

5. Chou CH, Bennett GN, San KY: Effect of modulated glucose uptake on high-level recombinant protein production in a dense Escherichia coli culture. Biotechnol Prog 1994, 10:644-647.

6. Death A, Ferenci T: Between feast and famine: endogenous inducer synthesis in the adaptation of Escherichia coli to growth with limiting carbohydrates. J Bacteriol 1994, 176:5101-5107.
7. Flores N, Flores S, Escalante A, de Anda R, Leal L, Malpica R, Georgellis D, Gosset G, Bolívar F: Adaptation for fast growth on glucose by differential expression of central carbon metabolism and gal regulon genes in an Escherichia coli strain lacking the phosphoenolpyruvate:carbohydrate phosphotransferase system. Metab Eng 2005, 7:70-87.

8. McDonald TP, Walmsley AR, Henderson PJF: Asparagine 394 in putative helix 11 of the galactose- $\mathrm{H}+$ symport protein (GalP) from Escherichia coli is associated with the internal binding site for cytochalasin B and sugar. J Biol Chem 1997, 272:15189-15199.

9. Lunin W, Li Y, Schrag JD, lannuzzi P, Cygler M, Matte A: Crystal structures of Escherichia coli ATP-dependent glucokinase and its complex with glucose. J Bacteriol 2004, 186:6915-6927.

10. Han $\mathrm{KH}$, Lim C, Hong J: Acetic acid formation in Escherichia coli fermentation. Biotechnol Bioeng 1992, 39:663-671.

11. Majewski RA, Domach MM: Simple constrained-optimization view of acetate overflow in E. coli. Biotechnol Bioeng 1990, 35:732-738.

12. Delgado J, Liao JC: Inverse flux analysis for reduction of acetate excretion in Escherichia coli. Biotechnol Prog 1997, 13:361-367.

13. Valgepea K, Adamberg K, Nahku R, Lahtvee PJ, Arike L, Vilu R: Systems biology approach reveals that overflow metabolism of acetate in Escherichia coli is triggered by carbon catabolite repression of acetyl-CoA synthetase. BMC Sys Biol 2010, 4:166.

14. Valgepea K, Adamberg K, Vilu R: Decrease of energy spilling in Escherichia coli continuous cultures with rising specific growth rate and carbon wasting. BMC Sys Biol 2011, 5:106.

15. Shiloach J, Kaufman J, Guillard AS, Fass R: Effect of glucose supply strategy on acetate accumulation, growth, and recombinant protein production by Escherichia coli BL21 (IXDE3) and Escherichia coli JM109. Biotechnol Bioeng 1996, 49:421-428.

16. Konstantinov K, Kishimoto M, Seki T, Yoshida T: A balanced DO-stat and its application to the control of acetic acid excretion by recombinant Escherichia coli. Biotechnol Bioeng 1990, 36:750-758.

17. Yang YT, San KY, Bennett GN: Metabolic flux analysis of $E$. coli deficient in the acetate production pathway and expressing the $B$. subtilis acetolactate synthase. Metab Eng 1999, 1:26-34.

18. De Anda R, Lara AR, Hernandez V, Hernandez-Montalvo V, Gosset G, Bolivar F, Ramirez OT: Replacement of the glucose phosphotransferase transport system by galactose permease reduces acetate accumulation and improves process performance of Escherichia coli for recombinant protein production without impairment of growth rate. Metab Eng 2006, 8:281-290.

19. Lara AR, Ramírez OT: Plasmid DNA production for therapeutic applications. In Recombinant Gene Expression. Methods in Molecular Biology. 3 Vol. 824, part 2nd edition. Edited by Lorence A. New York: Humana Press; 2012:271-303.

20. Soto R, Caspeta L, Barrón BL, Gosset G, Ramírez OT, Lara AR: High celldensity cultivation in batch mode for plasmid DNA vaccine production by a metabolically engineered $E$. coli strain with minimized overflow metabolism. Biochem Eng J 2011, 56(3):165-171.

21. Pablos TE, Soto R, Meza E, Le Borgne S, Gosset G, Ramírez OT, Lara AR: Enhanced production of plasmid DNA by engineered Escherichia coli strains. J Biotechnol 2012, 158:211-214.

22. Bachmann BJ: Pedigrees of some mutant strains of Escherichia coli K-12. Bacteriol Rev 1972, 36:525-557.

23. Datsenko KA, Wanner BL: One-step inactivation of chromosomal genes in Escherichia coli K-12 using PCR products. Proc Natl Acad Sci USA 2000, 97:6640-6645

24. Baba T, Ara T, Hasegawa M, Takai Y, Okumura Y, Baba M, Datsenko KA, Tomita M, Wanner BL, Mori H: Construction of Escherichia coli K-12 inframe, single-gene knockout mutants: the Keio collection. Mol Syst Biol 2006, 2:2006-2008

25. Herrera E, Barcenas P, Hernández R, Méndez A, Pérez-Ishiwara G, Barrón B: A 176 amino acid polypeptide derived from the mumps virus $\mathrm{HN}$ ectodomain shows immunological and biological properties similar to the HN protein. Virol J 2010, 7:1-11

26. Meza E, Becker J, Bolivar F, Gosset G, Wittmann C: Consequences of phosphoenolpyruvate:sugar phosphotranferase system and pyruvate kinase isozymes inactivation in central carbon metabolism flux distribution in Escherichia coli. Microb Cell Fact 2012, 11:127.

27. Borja GM, Meza-Mora E, Barrón B, Gosset G, Ramírez OT, Lara AR: Engineering Escherichia coli to increase plasmid DNA production in high cell-density cultivations in batch mode. Microb Cell Fact 2012, 11:132. 
28. Korner H, Sofia HJ, Zumft WG: Phylogeny of the bacterial superfamily of Crp-Fnr transcription regulators: exploiting the metabolic spectrum by controlling alternative gene programs. FEMS Microbiol Rev 2003, 27:559-592.

29. Kayser A, Weber J, Hecht V, Rinas U: Metabolic flux analysis of Escherichia coli in glucose-limited continuous culture. I. Growth-rate-dependent metabolic efficiency at steady state. Microbiology 2005, 151:693-706.

30. Picon A: Teixeira de Mattos MJ, Postma PW: Reducing the glucose uptake rate in Escherichia coli affects growth rate but not protein production. Biotechnol Bioeng 2005, 90:191-200.

31. Bäcklund E, Markland K, Larsson G: Cell engineering of Escherichia coli allows high cell density accumulation without fed-batch process control. Bioprocess Biosyst Eng 2008, 31:11-20.

32. Steinsiek S, Bettenbrock K: Glucose transport in Escherichia coli mutant strains with defects in sugar transport systems. J Bacteriol 2012, 194:5897-5908.

33. Bäcklund E, Ignatushchenko M, Larsson G: Suppressing glucose uptake and acetic acid production increases membrane protein overexpression in Escherichia coli. Microb Cell Fact 2011, 10:35.

34. Sauer U, Lasko DR, Fiaux J, Hochuli M, Glaser R, Szyperski T, Wüthrich K, Bailey JE: Metabolic flux ratio analysis of genetic and environmental modulations of Escherichia coli central carbon metabolism. J Bacteriol 1999, 181:6679-6688.

35. Williams JA, Carnes AE: Hodgson, CP: Plasmid DNA vaccine vector design: impact on efficacy, safety and upstream production. Biotechnol Adv 2009, 27:353-370

doi:10.1186/1475-2859-12-42

Cite this article as: Fuentes et al:: Modification of glucose import capacity in Escherichia coli: physiologic consequences and utility for improving DNA vaccine production. Microbial Cell Factories 2013 12:42.

\section{Submit your next manuscript to BioMed Central and take full advantage of:}

- Convenient online submission

- Thorough peer review

- No space constraints or color figure charges

- Immediate publication on acceptance

- Inclusion in PubMed, CAS, Scopus and Google Scholar

- Research which is freely available for redistribution 